\title{
COVID-19 and acute kidney injury in pediatric subjects: is there a place for eculizumab treatment?
}

\author{
Hernán Trimarchi ${ }^{1} \cdot$ Rosanna Coppo ${ }^{2}$
}

Published online: 29 September 2020

(C) Italian Society of Nephrology 2020

The coronavirus disease 2019 (COVID-19) pandemic is mostly affecting adult or elderly subjects often presenting with several co-morbidities [1]. The lack of children among the first cases described in Wuhan, China [2], and the mild disease reported in Chinese children in large series [3] have suggested that children may escape the major disease manifestations, although they can be infected. Since most cases are asymptomatic it is still unclear whether children are under-diagnosed or they are actually protected from viral invasion and overt disease. Despite these observations, recent collaborative studies have reported the development of multisystem inflammatory syndrome in children (MISC) in association with COVID-19, leading to serious and life-threatening illness [4]. Renal involvement with acute kidney injury (AKI) was detected in 5-10\% of these cases. These children were treated with immunomodulatory drugs, including intravenous (i.v.) immunoglobulins, glucocorticosteroids and interleukin (IL-6 or IL-1 receptor) inhibitors.

In the present issue, Mahajan et al. report a case of a 14-year-old girl with AKI associated to SARS-COV-2 infection, who developed laboratory features of thrombotic microangiopathy (TMA) and was successfully managed with the C5 inhibitor eculizumab [5]. The adolescent fulfilled the criteria for MIS-C [4]. As observed in one-third of cases with MIS-C, she had late positivity of IgG antibodies one week after initial symptoms, with negative COV-2 PCR. Inflammatory markers were elevated, respiratory distress needed intubation, myocardial and coronary involvement became manifest and dialysis-requiring AKI developed. The initial treatment choice was pragmatic and timely, including steroid pulses, i.v. immunoglobulins and the interleukin

Rosanna Coppo

rosanna.coppo@unito.it

1 Nephrology Service, Hospital Británico de Buenos Aires, Buenos Aires, Argentina

2 Fondazione Ricerca Molinette, Regina Margherita Hospital, Turin, Italy
1 receptor antagonist anakinra. However, while the MIS-C slightly improved, she developed features suggestive of complement-mediated TMA, with increased Cb5-9 levels. Noteworthy, plasma C3 and C4 levels were depressed since admission. Hence eculizumab was considered as a rescue therapy aimed at controlling the progressively worsening TMA. The patient improved after the first infusion, recovered her renal function and became gradually normotensive with normal urine analysis.

One of the reasons we found this case of particular interest is that it reminds us of a similar experience by one of the authors who observed a dramatic effect of eculizumab in a 4-year-old child with diffuse proliferative lupus nephritis who developed complement-mediated TMA and AKI [6]. She fully recovered but needed chronic eculizumab treatment for atypical hemolytic uremic syndrome (aHUS). Genetic studies were negative. C3 and C4 levels were deeply depressed even when aHUS and systemic lupus erythematosus (SLE) were under control. We diagnosed an association between the two diseases, which was recently defined as an independent clinical entity [7]. The case reported by Mahajan et al. belongs to complement-mediated non-lupus TMA, despite negative genetic tests for aHUS, as confirmed by a favorable effect of $\mathrm{C} 5$ inhibition. In both cases the initial disease was a severe multisystem inflammatory syndrome due to SARS-CoV-2 or systemic lupus erythematosus. In both children the anti-inflammatory and immunosuppressive therapy only partially controlled the symptoms, being unable to halt a critical worsening of clinical conditions and renal failure mostly due to TMA. Eculizumab, a humanized monoclonal antibody with high-affinity binding to $\mathrm{C} 5$, hence blocking the generation of C5-9 complex is nowadays the treatment of choice for aHUS [8].

Complement activation plays a crucial role in SARS$\mathrm{CoV}-2$ infection and renal damage. The viral binding to its receptor angiotensin-converting enzyme 2 (ACE2) highly expressed on endothelial cells, triggers cytokine release. The viral $\mathrm{S}$ glycoprotein binds to mannose binding lectin (MBL) 
and mannose associated serine protease (MASP2) activating the lectin complement pathway, favoring a positive feedback loop which leads to sustained alternative pathway activation, thus inducing inflammation and concurrent activation of the coagulation cascade [8].

Therefore, there is a potential pathophysiologic rationale for the use of eculizumab in severe SARS-CoV-2 infections that present with TMA.

Eculizumab does not however qualify as a miraculous drug in SARS-CoV-2 infection, as another recent case described by one of the authors demonstrates. This patient was a 24-year-old male with a six-year history of a kidney graft due to a pretransplant diagnosis of aHUS (no complement molecule-associated mutation was identified), who was chronically treated with a maintenance dose of eculizumab ( $900 \mathrm{mg}$ bi-weekly) associated with methylprednisone $4 \mathrm{mg}$ / day, mycophenolate $720 \mathrm{mg} /$ day, monthly $500 \mathrm{mg}$ belatacept (a selective T-lymphocyte co-stimulation blocker). He was admitted with severe diffuse bilateral SARS-CoV-2 pneumonia [9] and elevated D-dimer and fibrinogen concentrations, suggesting a pro-coagulant state due to pulmonary microthrombosis, as described in autopsies of subjects with COVID-19 infections [1], despite ongoing chronic eculizumab treatment. On admission to intensive care he received i.v. dexamethasone $6 \mathrm{mg} /$ day and $200 \mathrm{~mL}$ convalescent plasma infusion. Oxygen was administered via mask reservoir $(5 \mathrm{~L} / \mathrm{min})$ and vigil pronation cycles without any need for mechanical ventilation. Two weeks after admission, the scheduled eculizumab and belatacept infusions were administered and the patient was discharged [9]. This case combined aHUS-associated predisposition to microvascular injury and chronic immunosuppression. Eculizumab administration, both chronic and early in the course of COVID-19 did not prevent the severe endothelial cell injury, as assessed by a 36-fold increase in D-dimer. We supposed that the sudden increase in thrombophilic state due to SARS-CoV-2 infection suddenly increased the needed amount of eculizumab, which was unable to completely block the complement cascade, leaving open the possibility of some benefits of the drug in the rapid resolution of the severe pulmonary involvement.

According to clinicaltrials.gov, there are seven ongoing clinical trials exploring six different anti-complement drugs for COVID-19. We believe that the case report published by Mahajan et al. is relevant not only because the results were straightforward, but also because it underscores the complement system as a main target of SARS-CoV-2. It remains to be clarified whether complement activation and the severe endothelial injury, leading to the development of TMA, were consequences of the cytokine storm typical of MIS-C, or if SARS-COV-2 unmasked an underlying primary aHUS yet to be confirmed by genetic testing (even though, as mentioned above, negative genetic result does not rule out primary aHUS). The follow-up of this case, with persistent normalization of clinical and laboratory data or subsequent relapses, will be helpful in discriminating the possibility of COVID-19 mediated-TMA and/or covert aHUS. However, while waiting for further follow-up, the present report deserves our utmost interest because it highlights the role of the complement system activation in SARS-COV-2 infection and the pharmacological interventions to attenuate the micro-thrombotic complications associated with COVID-19.

\section{References}

1. Zhou F, Yu T, Du R et al (2020) Clinical course and risk factors for mortality of adult inpatients with COVID-19 in Wuhan, China: a retrospective cohort study. The Lancet 395:1054-1062

2. Liu W, Zhang Q, Chen J et al (2020) Detection of COVID-19 in children in early January 2020 in Wuhan China. N Engl J Med 382(14):1370-1371. https://doi.org/10.1056/NEJMc2003717

3. Shen K, Yang Y, Wang T et al (2020) Diagnosis, treatment, and prevention of 2019 novel coronavirus infection in children: experts' consensus statement. World J Pediatr 16(3):223-231. https://doi.org/10.1007/s12519-020-00343-7

4. Feldstein LR, Rose EB, Horwitz SM et al (2020) Multisystem inflammatory syndrome in US children and adolescents. N Engl J Med 383(4):334-346. https://doi.org/10.1056/NEJMoa2021680

5. Mahajan R, Lipton M, Broglie L, Jain NG, Uy NS (2020) Eculizumab treatment for renal failure in a pediatric patient with COVID-19. J Nephrol. https://doi.org/10.1007/s40620-020-00858 $-2$

6. Coppo R, Peruzzi L, Amore A et al (2015) Dramatic effects of eculizumab in a child with diffuse proliferative lupus nephritis resistant to conventional therapy. Pediatr Nephrol 30(1):167-172. https://doi.org/10.1007/s00467-014-2944-y

7. Park MH, Caselman N, Ulmer S, Weitz IC (2018) Complementmediated thrombotic microangiopathy associated with lupus nephritis. Blood Adv 2(16):2090-2094. https://doi.org/10.1182/ bloodadvances.2018019596

8. Loirat C, Frémeaux-Bacchi V (2011) Atypical hemolytic uremic syndromeOrphanet. J Rare Dis 6:60. https://doi.org/10.1186/17501172-6-60(Published online 2011 Sep 8)

9. Trimarchi GR, Lampo M, Monkowski M, Lodolo MJ (2020) Eculizumab, SARS-COV-2 and atypical hemolytic uremic syndrome. Clin Kidney J. https://doi.org/10.1093/ckj/sfaa166 (In press)

Publisher's Note Springer Nature remains neutral with regard to jurisdictional claims in published maps and institutional affiliations. 